\title{
Validation of the International Reading Speed Texts in a Canadian Sample
}

\author{
Elliott Morrice ${ }^{1,2, *}$, Julian Hughes ${ }^{1}$, Zoey Stark ${ }^{1}$, Walter Wittich ${ }^{1,2,3,4}$, and Aaron \\ Johnson $^{1,2}$
}

*Corresponding author

E-mail: elliott.morrice@mail.concordia.ca

${ }^{1}$ Concordia University, Department of Psychology, Montreal, Quebec, H4B 1R6, Canada

${ }^{2}$ CRIR/ Centre de réadaptation LETHBRIDGE-LAYTON-MACKAY Rehabilitation Centre du Centre Intégré Universitaire de Santé et Services Sociaux (CIUSSS) Centre ouest de l'ile de Montréal, Québec, H4B 1R3, Canada

${ }^{3}$ École d'optométrie, Université de Montréal, Montréal, Québec, H3T 1P1, Canada

${ }^{4}$ CRIR/Institut Nazareth et Louis-Braille du CISSS de la Montérégie-Centre, Longueuil, Québec, J4K 5G4, Canada 
IREST CANADIAN VALIDATION

\section{Structured Abstract}

Purpose: The purpose of these studies are (1) to validate the IReST in an English-speaking Canadian sample; and (2) examine how reading comprehension questions and reduced visual acuity effect reading speed on the IReST.

Materials \& Methods: Study 1: Canadian English speakers $(n=25)$ read all 10 IReST following the procedures used in the original IReST validation. Study 2: Canadian English speakers $(n=50)$ read all $10 \mathrm{IReST}$, half with normal/corrected-to-normal vision and half with reduced visual acuity, and were asked reading comprehension questions.

Results: No significant differences were found between Canadian sample and the published IReST values (in all cases $p>.05, M_{\text {diff }}=[-5.30,11.43]$, Cohen's $d=[-.15, .27]$, Bayes Factors $=[0.41,0.09])$. Assessing reading comprehension with multiple choice questions on the IReST significantly reduced reading speeds in the normal vision condition $\left(M_{\text {diff }}=25.3,95 \%\right.$ $\mathrm{CI}=[-16.7,-34.1])$ and in the simulated impairment condition $\left(M_{\text {diff }}=59.3,95 \% \mathrm{CI}[-47.7,-71]\right)$. Conclusions: The IReST is a valid measure that can be used to assess reading speed in a Canadian English speaking sample. If researchers/clinicians wish to assess both reading speed and comprehension, using multiple choice reading comprehension questions, then the values provided by the IReST will likely overestimate an individual's true reading speed in individuals with normal/corrected-to-normal vision and reduced visual acuity.

Keywords: IReST, Validation, Reading Comprehension, Low Vision, Low Vision Rehabilitation 
IREST CANADIAN VALIDATION

\section{Acknowledgements}

1. This work was supported in part by the Vision Research Network, the Fonds de recherche du Québéc - Santé (\#30620 \& \#32643), and by the Social Sciences and Humanities Research Council of Canada - Canada Graduate Scholarships-Master's Program (CGS-M).

2. We would like to thank Natalie Phillips for her input and advice in the conceptual design of this experiment.

\section{Declaration of Interest}

The authors report no conflicts of interest.

\section{Data Availability Statement}

The data and analysis used in this article are available on the OSF at https://osf.io/7ad9g/ 
IREST CANADIAN VALIDATION

\section{Introduction}

The International Reading Speed Texts (IReST) is a standardized measure to assess continuous reading[1,2]. The English language IReST was developed for British English (UK), and is used throughout the United States and Canada to assess reading speed [e.g., 3-5]. However, the English language IReST has not been validated in a North American sample. Additionally, the IReST is frequently used by low vision rehabilitation specialists to assess both reading speed and comprehension in individuals with low vision; yet there have been no studies that have examined the impact of assessing reading comprehension using the IReST. While some studies have used to IReST to examine how behavioral training or assistive technology devices, e.g., eccentric viewing or magnifiers, can improve reading in individuals with visual impairments, there are no studies that have examined the overall impact of visual impairments on mean reading speeds on the IReST[1-7]. Therefore, the purpose of the two studies presented here is (1) to validate the IReST in an English-speaking Canadian Sample; (2) determine the impact of assessing reading comprehension questions on the IReST in a sample of individuals with normal vision and with simulated impairments.

\section{Study 1: Validation of the IReST in a Canadian Sample}

The IReST was developed in Europe, and has been translated, linguistically adapted, and validated in 17 different languages[1,2]. Each IReST provides a measure of mean reading speed (words per minute) and variance (standard deviation) that allows for the comparison of an individual's reading speed to the validated reading speeds provided by the IReST manufacturer. As the English language IReST was developed for British English, it uses sentence structure, grammar, syntax, terms, and phrases that may be less familiar to North American English speakers, e.g., the terms greengrocer, felling, gnaws, recourse. These factors may negatively 


\section{IREST CANADIAN VALIDATION}

impact the reading speeds on the IReST. Indeed, previous researchers have suggested that there may be significant differences in individuals reading speeds depending on their country/region of origin, e.g., differences in reading rates have been found between individuals from Wisconsin versus those from North Carolina[8,9]. One reason there may be differences in the reading speeds of Canadians compared to the values provided by the IReST is the proportion of bilinguals in the Canadian population[10]. Researchers have shown that while no differences exist between monolingual and bilingual L1 (first/dominant language) readers, bilingual L2 (second language) readers read significantly slower than monolinguals[11]. These differences in reading speed are believed to be due to discrepancies in language proficiency/automaticity and this may contribute to regional differences in reading speed[12-15]. However, the original IReST publication does not report any data related to the language background or proficiency, e.g., self-reported reading proficiency, of its English-speaking sample. Thus, for the purpose of this study it will be assumed that participants in the original IReST validation were either monolingual, or L1 English speakers. As data for the present study were collected in Montreal, a city with a high proportion of bilinguals/trilinguals, participants in this study were restricted to L1 English speakers to control for these differences.

This study examined whether the normative values of the IReST are valid in an L1 English-speaking Canadian sample, and it was hypothesized that the reading speeds of Canadian English speakers would fall outside of the standards established by the UK English language IReST[1,2]. In this experiment, normally sighted L1 English speakers were asked to read all 10 English language IReST aloud following the same procedures used in the original IReST validation experiment and the published IReST administration protocols.

\section{Methods}


IREST CANADIAN VALIDATION

The research followed the tenets of the Declaration of Helsinki, informed consent was obtained from the subjects after explanation of the nature and possible consequences of the study. The research protocol for study 1 and 2 was approved by the human research ethics committee of Concordia University (certificate 30003975), in accordance with the Canadian Tri-Council Policy Statement of ethical conduct for research involving humans[16].

\section{Participants}

Twenty-five undergraduate students (21 females), between the ages of 19 and $41(\mathrm{M}=21.72$, $\mathrm{SD}=4.94$ ), were recruited from Concordia University. Participants were required to have normal/corrected-to-normal vision (20/20 [6/6] or better - verified using the Freiburg Visual Acuity and Contrast Sensitivity Test[17] (FrACT)), and be L1 English speakers (verified using the Concordia University Language Background Questionnaire[3]). No participants reported reading or attention disabilities. A sample size of 25 participants was used to match the sample size used in the original IReST validation experiment[1].

\section{Measures}

\section{Concordia University Language Background Questionnaire}

The Concordia University Language Background Questionnaire[3] was used to obtain demographic and language background information. Data were collected on participants' first, second, and third languages (as applicable), as well as self-reported speaking, reading, writing, and listening ability in English, French, and other languages. Participants self-reported any visual/hearing impairments, and any known reading or attention disabilities.

\section{Reading Speed}

Following the original IReST protocol[1], participants were asked to read the IReST aloud, while the researcher recorded any errors they made on a separate printed copy. Reading speed was 
IREST CANADIAN VALIDATION

measured using a stopwatch that was started when participant began to read the first word of each text, and was stopped when they read the last word. Reading speed, in words per minute (wpm), was calculated using the formula $(c w / s) * 60=w p m$, where $c w$ is the number of words read correctly, and $s$ is the amount of time it took to read each text in seconds (rounded to the nearest $1 / 10$ th of a second).

\section{Freiburg Visual Acuity and Contrast Sensitivity Test (FrACT)}

The FrACT is a computer-based test that assesses visual acuity and contrast sensitivity[17], it is a valid and reliable measure of visual acuity when compared to the ETDRS charts[18], and has been shown to have adequate test-retest reliability[19]. The FrACT was presented on an Apple iMac (21 inch - 2015 model) at a seated distance of $140 \mathrm{~cm}$, and participants were asked to complete both measures of visual acuity and contrast sensitivity.

\section{Procedure}

Upon arrival, participants were instructed on the content/procedure of the study and informed consent was obtained. Participants completed the Concordia University Language Background Questionnaire, and measures of their visual acuity and contrast sensitivity were obtained using the FrACT. The same procedure that was used in the original IReST validation experiment $[1,2]$ was used in the current study, with the exception that in this study the order in which the IReST were read was counterbalanced to reduce practice effects, whereas in the original study the order of text presentation was randomized. The IReST were presented to participants at a distance of $40 \mathrm{~cm}$, and before reading each text they were asked to "read the text aloud as quickly as possible and without going back and making corrections." In line with the original study, a stopwatch was used to measure reading time and incorrectly read or omitted words were counted. In the original study, the authors report that error counts were not used in the calculation of mean reading speed 
IREST CANADIAN VALIDATION

due to incomplete documentation across languages, and the rarity with which errors occurred. In this study errors/omissions were used in the calculations of mean reading speed following the administration protocols provided by the IReST manufacturer. The protocol states that misread/omitted words should be noted and subtracted from the total number of words in each text. The average number of misread/omitted words across all 10 texts was 1.34 words $(S D=1.39)$.

\section{Data Analysis}

To determine whether the normative values of the IReST are valid in an English- speaking Canadian sample, difference scores (in wpm) between the Canadian sample and IReST values were examined using multiple one-sample t-tests. For each analysis, the alternative hypothesis specified that the mean was less than 0, e.g., $H_{1}: M_{I R e S T I}<\mu_{I R e S T I}$. Analyses were computed using both traditional null hypothesis significance testing and Bayes factors; a Bayes factor allows for the interpretation of null results as either insufficient evidence to make a conclusion, or as finding evidence for the null hypothesis[20,21]. Bayes factors $(B F)$ were calculated using Jamovi $1.0[22]$, and for each analysis a Cauchy prior width of 0.707 was used. $\mathrm{A} \mathrm{BF}_{10}$ is the strength of evidence for the research hypothesis, and the $\mathrm{BF}_{01}$ is the strength of evidence for the null hypothesis. For each analysis, the robustness of the posterior was checked using wider priors of 1.00 and 1.50 to ensure that the choice of prior distribution did not significantly impact the results. The $95 \%$ confidence intervals around the mean difference were used as a measure of the margin of uncertainty around the estimated difference between the two means, and Cohen's $d$ was used as a measure of effect size.

\section{Results}

\section{Descriptive Statistics}


IREST CANADIAN VALIDATION

All participants' first and dominant language was English, 98\% of participants were bi-lingual, and $60 \%$ of participants were tri-lingual. See table 1 for a breakdown of participants selfreported language fluency (speaking, reading, writing, and listening ability) in English, French, and other languages. Participants had a mean visual acuity of -0.11 logMAR (20/15.5Snellen; $\mathrm{M}=-0.11, \mathrm{SD}=0.10, \mathrm{CI}[-0.16,-.07])$, and a mean contrast sensitivity of $2.01 \log \mathrm{CS}(\mathrm{M}=2.01$, $\mathrm{SD}=0.19, \mathrm{CI}[1.94,2.09])$.

[Table 1 near here]

\section{Canadian Sample Compared to UK IReST Values}

Results of the one-sample t-tests indicate that there are no statistically significant differences between the Canadian samples mean reading speed on the IReST, and the values provided by the IReST (table 2). In all cases, p-values were greater than 0.05. Mean difference scores ranged from $-5.30 \mathrm{wpm}$ to $11.43 \mathrm{wpm}$, with $95 \%$ confidence intervals ranging from -17.36 to 25.76 (Figure 1). Measures of effect size using Cohen's $d$ ranged from -0.15 to 0.27 , with $95 \%$ confidence intervals overlapping 0 , and ranging from -0.48 to 0.60 . Bayes factors $\left(B F_{10}\right.$ 's) ranged from 0.41 to 0.09 ; such that the probability of the null hypothesis that there is no difference between reading speeds between the samples was 2.41 to 10.18 times greater than the alternative hypothesis, i.e., the Canadian sample would read slower than the published IReST values.

[Table 2 near here]

[Figure 1 near here]

\section{Discussion}

The primary purpose of study 1 was to investigate whether the normative values of the IReST established with UK readers could be replicated in an L1 English-speaking Canadian sample. As 
IREST CANADIAN VALIDATION

the mean reading speeds of the Canadian sample fell within the standards established by the UK English language IReST, i.e., participants in the Canadian sample read at the same rate, the values provided by the IReST appear to be valid in an L1 English-speaking Canadian sample. While there were no significant differences in reading speeds between samples, it should be noted that there may be greater variability in the reading speeds of the Canadian sample compared to the standards of the IReST. Measures of variability in reading speed provided by the IReST, i.e., standard deviations, range from 22-32, whereas the standard deviations of the Canadian sample range from 31.80-44.83. It is conceivable that the higher variability in reading speeds in the Canadian sample may be due to the high proportion of bilinguals (98\%) and trilinguals $(60 \%)$ included in this sample, even though there were no differences in overall mean reading speeds[11-15].

\section{Study 2: Impact of Reading Comprehension and Reduced Visual Acuity on the IReST}

One of the most frequently cited reasons individuals seek low vision rehabilitation (LVR) is difficulty reading[23]. LVR specialists assist patients by providing behavioral training and assistive technology devices aimed at improving reading speed and comprehension[24-26]. To accomplish this goal, LVR specialists require standardized measures that can be used to determine which training/devices optimally improve reading. While the IReST provides a standardized measure of reading speed, it lacks a measure of reading ability, such as a reading comprehension question. Researchers have shown that reading for speed compared to reading for comprehension engages different reading processes (e.g., see Carver[27] literature), and results in overall slower reading speeds. Consequently, if the values provided by the IReST are used as points of comparison when assessing reading comprehension in the context of LVR, then they may overestimate an individual's true reading speed. Assessing reading comprehension in 


\section{IREST CANADIAN VALIDATION}

individuals with visual impairments is an important component of LVR, as literacy has been shown to be a strong predictor of health self-management in older adults [28-30]. Therefore, the purpose of this study was to examine the impact of assessing reading comprehension, through the use of multiple-choice questions, on reading speed on the IReST in a sample of individuals with normal/corrected-to-normal vision and simulated visual impairments (simulated reductions in visual acuity and contrast sensitivity).

Simulated reductions in visual acuity and contrast sensitivity were used in lieu of a sample of individuals with actual visual impairments due to the heterogeneity of visual impairments[31-33]. More specifically, individuals with visual impairments can have varying degrees of visual acuity and contrast sensitivity, depending on the type and severity of their impairment(s) and may have varying levels of reading ability depending on how well they have adapted to their condition[34,35]. An advantage of using a simulated visual impairment is the uniform reduction in visual acuity and contrast sensitivity across participants, to determine the general impact of low visual acuity (here defined as 20/80 [6/24]) on reading). Conversely, a limitation of using a simulated visual impairment is that the simulation may underestimate this population's true reading speed, because individuals with low vision typically have adapted somewhat to their impairments, or may have developed compensatory techniques to improve their reading speed[34,35]. Using a simulated visual impairment does not take these compensatory strategies into account, as normally sighted individuals will not have had enough time to adapt.

It was hypothesized that (1) assessing reading comprehension on the IReST would significantly reduce participants' reading speeds; and (2) that the combined impact of assessing 
IREST CANADIAN VALIDATION

reading comprehension in individuals with a simulated reduction in visual acuity and contrast sensitivity would result in further reductions in reading speed on the IReST.

\section{Methods}

\section{Participants}

Fifty undergraduate students $(n=50 ; 42$ females), between the ages of 18 and $45(M=24.06$, $\mathrm{SD}=6.45)$, were recruited from Concordia University. Participants were required to have normal/corrected-to-normal vision (20/20 [6/6] or better - verified using the FrACT[17]), and be L1 English speakers (verified using the Concordia University Language Background Questionnaire[3]). Six participants reported having reading or attention disabilities and consequently analyses were conducted both including and excluding their data. However, this did not result in any significant differences, therefore their data were retained.

\section{Measures}

Study 2 used the same measures as described in Study 1; the Concordia University Language Background Questionnaire, the IReST, and the FrACT, in addition to the low vision simulator goggles described below.

Fork in the Road Goggles (20/80 [6/24]), WI, USA

Low vision simulation goggles[36] were used to simulate a visual acuity of 20/80 (6/24 or 0.6 $\log$ MAR) in the simulated impairment condition. These goggles have lenses that are designed to simulate a visual impairment analogous to a cataract, with individuals experiencing a general reduction in visual acuity and contrast sensitivity across the visual field. This was verified for all participants (see procedure below). In the normal vision condition, participants wore the goggles with the lenses removed.

\section{Procedure}




\section{IREST CANADIAN VALIDATION}

Upon arrival, participants were instructed on the content/procedure of the study and informed consent was obtained. Participants completed the Concordia University Language Background

Questionnaire, were systematically assigned to a counterbalanced order of testing conditions, and were asked to read all 10 IReST aloud: 5 with their normal vision and 5 with a simulated impairment. The Reading Comprehension (RC; normal vision condition) and Reading Comprehension with Impairment (RCI; impaired vision condition) groups were counterbalanced such that the first set of 5 IReST were read with either the participant's normal/corrected-tonormal vision, or with the simulated 20/80 visual impairment. The order in which the IReST were read was also counterbalanced to reduce learning effects and fatigue.

In the $\mathrm{RC}$ condition, participants wore the goggles without lenses, and baseline measures of participant's visual acuity and contrast sensitivity were obtained using the FrACT. Five of the IReST were then presented to the participants at a distance of $40 \mathrm{~cm}$, and they were instructed to "read each text aloud as quickly as possible and without going back and making corrections." After reading each text, participants were asked a multiple-choice reading comprehension question ( 3 options, only one correct). Participants then read the remaining 5 IReST in the RCI condition.

In the RCI condition, participants wore the goggles with the 20/80 low vision simulator lenses. Participant's visual acuity and contrast sensitivity were measured before reading the remaining IReST to ensure the goggles were reducing acuity and contrast sensitivity. In the RCI condition participants were permitted to hold the texts as near as required to facilitate reading, as stipulated by the IReST administration protocol for assessing individuals with visual impairments, and the reading distance was recorded. After reading the 5 texts, another measure of visual acuity and contrast sensitivity was obtained to ensure that participants did not adapt to 
IREST CANADIAN VALIDATION

the simulator goggles. If participants completed the RCI condition first, the same procedure was followed in reverse.

\section{Data Analysis}

Both a traditional and a Bayesian analysis of variance (ANOVA) were used as a manipulation check to determine if the goggles significantly reduced participant's visual acuity/contrast sensitivity in the impairment condition, and whether participants adapted to the goggles. Mean difference scores were calculated between the Canadian Sample's (CS) mean reading speed on the IReST in study 1 , and the mean reading speeds of the RC and RCI conditions. A traditional and a Bayesian paired-sample t-test were used to determine whether there were significant differences between the RC/RCI conditions. The 95\% confidence intervals around the mean difference scores were used to determine whether there were significant differences between the $\mathrm{CS}, \mathrm{RC}$, and RCI conditions; i.e., if the confidence intervals do not capture 0 , this suggests there is a significant difference between the $\mathrm{CS}, \mathrm{RC}$, and $\mathrm{RCI}$ conditions. For each Bayes factor, the robustness of the posterior was checked using wider priors of 1.00 and 1.50 to ensure that the choice of prior distribution did not significantly impact the results. Two scores represented extreme outliers and were replaced with the next highest values; this did not significantly change the results.

\section{Results}

\section{Descriptive Statistics}

All participants' first and dominant language was English, all participants were bi-lingual, and $54 \%$ of participants were tri-lingual. See table 1 for a breakdown of participants self-reported language fluency (speaking, reading, writing, and listening ability) in English, French, and other languages. 
IREST CANADIAN VALIDATION

\section{Visual Acuity and Low Vision Simulator Goggles}

Participants' visual acuity and contrast sensitivity were assessed at three time-points: at baseline, before reading the texts in the RCI, and after reading the texts in the RCI condition. At baseline participants had a mean visual acuity of $-0.11 \log$ MAR (20/15.5 Snellen; $M=-0.11, \mathrm{SD}=0.09$, $95 \% \mathrm{CI}[-0.13,-.09])$ and a mean contrast sensitivity of $2.01 \log \mathrm{CS}(\mathrm{M}=2.01, \mathrm{SD}=0.21,95 \% \mathrm{CI}$ $[1.95,2.06])$. The low vision simulator goggles failed to produce the $20 / 80$ visual impairment they were designed to simulate; however, they were successful at simulating a visual impairment of 20/60, or 0.50 logMAR, which is the threshold in Quebec to be eligible to receive low vision rehabilitation services[37]. At the second time-point, the goggles simulated a visual acuity of $0.51 \log$ MAR (20/64 Snellen; $\mathrm{M}=0.51, \mathrm{SD}=0.16,95 \% \mathrm{CI}[0.46,0.55])$ and contrast sensitivity of $0.93 \log \mathrm{CS}(\mathrm{M}=0.93, \mathrm{SD}=0.28,95 \% \mathrm{CI}[0.85,1.01])$. At the third time-point the goggles simulated a visual acuity of $0.49 \log \operatorname{Mar}(20 / 61$ Snellen; $M=0.49, S D=0.17,95 \% C I[0.44,0.54])$ and contrast sensitivity of $0.96 \log C S(M=0.96, S D=0.18,95 \% C I[0.91,1.01])$.

Both traditional and Bayesian one-way ANOVA's using the Tukey correction for multiple post-hoc comparisons was conducted to compare the effect of the impairment goggles on visual acuity: results show there was a significant effect of impairment goggles on visual acuity $\left(F(2,147)=308.6, p<.001, \omega^{2}=0.80, B F_{10}=4.853 \times 10^{49}\right)$. The Bayes factor indicated that the probability of the research hypothesis, i.e., that there was a difference in measures of visual acuity, is $4.853 \times 10^{49}$ times greater than the probability of the null hypothesis. Post hoc comparisons indicated that there were statistically significant differences between baseline measures of visual acuity and measures taken at the second $\left(M_{d i f f}=-.62, s=0.03, p<.001, d=\right.$ 4.89, $\left.B F_{10}=6.127 \times 10^{39}\right)$ and third $\left(M_{d i f f}=-0.60, s=0.03, p<.001, d=-4.51, B F_{10}=7.987 \times 10^{36}\right)$ time-points. However, these differences were not significant between measures of visual acuity 
IREST CANADIAN VALIDATION

taken at time-points two and three $\left(M_{\text {diff }}=0.02, s=0.03, p=.80, d=0.11, B F_{10}=0.242\right)$, such that participant experienced the same simulated reduction in visual acuity across the experiment.

Both traditional and Bayesian one-way ANOVA using the Tukey correction for multiple post-hoc comparisons was conducted to compare the effect of the impairment goggles on contrast sensitivity: results show there was a significant effect of impairment goggles on contrast sensitivity $\left(F(2,147)=366.4, p<.001, \omega^{2}=0.83, B F_{10}=1.368 \times 10^{54}\right)$. The Bayes factor indicated that the probability of the research hypothesis, i.e., that there is a difference in measures of contrast sensitivity, was $1.368 \times 10^{54}$ times greater than the probability of the null hypothesis. Post hoc comparisons found there were significant decreases in contrast sensitivity between baseline and measures taken at the second $\left(M_{\text {diff }}=1.08, s=0.05, p<.001, d=4.35, B F_{10}=\right.$ $\left.4.851 \times 10^{35}\right)$ and third $\left(M_{d i f f}=1.05, s=0.05, p<.001, d=5.37, B F_{10}=1.596 \times 10^{43}\right)$ time-point. However, there were no significant differences between measures of contrast sensitivity taken at time-points two and three $\left(M_{\text {diff }}=-0.04, s=0.05, p=.78, d=-0.13, B F_{10}=0.255\right)$, such that participant experienced the same simulated reduction in contrast sensitivity across the experiment.

\section{Comparisons Between Canadian, RC, and the RCI Conditions}

To determine whether participants in the RC and RCI conditions would read the IReST significantly slower than the Canadian IReST values established in study 1 , mean difference scores were calculated for the RC/RCI conditions (see table 3). A traditional and Bayesian paired-sample t-test was conducted to determine whether there were significant differences between the RC/RCI conditions. The traditional NHST paired-sample t-test found there was a significant difference $\left(t(49)=9.37, p<.001, M_{\text {diff }}=34, d=1.32, B F_{10}=1.02 \times 10^{10}\right)$ between the RC $(M=-25.3, S D=30.6,95 \% C I[-16.70,-34.10])$ and the RCI conditions $(M=-59.3 S D=41,95 \% C I$ 
IREST CANADIAN VALIDATION

$[-47.75,71.05])$, such that asking multiple choice reading comprehension questions in the RCI condition resulted in significantly slower reading speeds compared to the RC condition. The Bayes factor indicated that the probability of the research hypothesis, i.e., that reading speeds would be slower in the RC and RCI conditions, was $1.02 \times 10^{10}$ times greater than the probability of the null hypothesis. According to the Bayes factor interpretation guidelines provided by Wetzels et al. (2011), a $B F_{10}$ of greater than 100 indicates "decisive evidence" for the research hypothesis[21].

[Table 3 near here]

As both the $95 \%$ confidence intervals around the mean difference in the RC and RCI conditions did not capture 0 , it can also be concluded that there was a significant difference between the both the $\mathrm{RC} / \mathrm{RCI}$ conditions and the Canadian sample from study 1 . Such that participants in the RC condition read on average 25.3 WPM slower than the Canadian sample, whereas participants in the RCI read on average 59.3 WPM slower than the Canadian sample.

\section{Discussion}

The purpose of this study was to examine how simulated visual impairment affects reading speed and text comprehension in individuals with normal/corrected-to-normal vision. This was achieved by the use of multiple-choice questions. As was hypothesized, we found that asking participants reading comprehension questions on the IReST significantly reduced reading speeds by an average of 25.3 WPM in the normal vision condition (see Figure 2). This is consistent with the Carver literature[27] that shows reading for comprehension results in slower reading and engages different reading processes compared to reading for speed. Taking reading comprehension into account is an essential component of LVR as previous research has shown that literacy in older adult is a strong predictor of health self-management; e.g., choosing a 
IREST CANADIAN VALIDATION

healthcare facility, monitoring a medical condition, and managing prescription medications[2830]. Therefore, when using the IReST to assess both reading speed and comprehension LVR specialists should use the IReST values from the RC condition presented in table 4. As hypothesized in the simulated impairment condition, it was found that assessing reading comprehension while participants had simulated reductions in visual acuity and contrast sensitivity significantly decreased reading speeds on average by a further 33.8 WPM (mean difference between the Canadian sample and the RCI sample was 59.39 WPM).

[Table 4 near here]

[Figure 2 near here]

\section{Conclusion}

The purpose of the two studies presented here was to: (1) validate the IReST in an Englishspeaking Canadian Sample; (2) determine the impact of assessing reading comprehension questions on the IReST in a sample of individuals with normal vision and with simulated impairments. Mean reading speeds of participants in Study 1 fell within the values provided by the IReST, therefore, the IReST appears to be a valid measure of reading speed in a Canadian sample. However, using the IReST to assess reading speed and comprehension, through the use of a multiple-choice reading comprehension question, significantly reduced reading speeds. Additionally, assessing reading comprehension while participants experienced a simulated reduction in visual acuity and contrast sensitivity further reduced reading speeds.

A limitation of the second study is the use of a simulated visual impairment. The low vision simulator goggles used in this study resulted in a uniform decrease in visual acuity and contrast sensitivity across the visual field; however, impairments caused by actual visual impairments, e.g., age-related macular degeneration (AMD), do not cause uniform impairments 
IREST CANADIAN VALIDATION

across the visual field, across individuals[38]. Previous research has shown that AMD can severely impact reading performance and require entirely new eye movement strategies unique to each individual[6,7,39,40]. Whereas, glaucoma may not impair an individual's visual acuity but still affect their reading performance[41-43]. Therefore, future studies should examine the impact of actual visual impairments, e.g., age-related macular degeneration, cataracts, glaucoma, on the IReST to determine what "normal" reading speeds may resemble in these populations. In clinical settings, LVR specialists should use the standardized values provided by the IReST as points of comparison when simply assessing reading speed; however, if they desire to assess both reading speed and comprehension, through the use of multiple choice reading comprehension questions, then they should use the values presented in table 4 . Finally, the results presented here suggest that assessing for reading comprehension on the IReST in individuals with sensory impairments will also result in further reductions in reading speed. As reading performance is determined by the type and severity of individuals impairment, e.g., central scotoma versus cataracts, researchers and clinicians may use the values obtained in this study as a rough estimate for expected reading speeds on the IReST before receiving LVR services.

\section{References}

1. Trauzettel-Klosinski S, Dietz K. Standardized assessment of reading performance: The new international reading speed texts IReST. Investig Ophthalmol Vis Sci. 2012;53:54525461.

2. Hahn G A, Penka D, Gehrlich C, Messias A, Weismann M, Hyvärinen L, Leinonen M, Feely M, Rubin G, Dauxerre C, et al. New standardised texts for assessing reading 


\section{IREST CANADIAN VALIDATION}

performance in four European languages. Br J Ophthalmol. 2006;90:480-484.

3. Morrice E, Johnson AP, Marinier J-A, Wittich W. Assessment of the Apple iPad as a lowvision reading aid. Eye [Internet]. 2017 February 3. Available from:

http://dx.doi.org/10.1038/eye.2016.309

4. Ramulu PY, Swenor BK, Jefferys JL, Rubin GS. Description and validation of a test to evaluate sustained silent reading. Invest Ophthalmol Vis Sci [Internet]. 2013 January 23;54:673-80. Available from:

http://iovs.arvojournals.org/article.aspx?doi=10.1167/iovs.12-10617

5. Swenor BK, Varadaraj V, Dave P, West SK, Rubin GS, Ramulu PY. Impact of the ability to divide attention on reading performance in glaucoma. Investig Ophthalmol Vis Sci. 2017;58:2456-2462.

6. Rubin G, Feely M. The Role of Eye Movements During Reading in Patients with AgeRelated Macular Degeneration (AMD). 2009:120-126. Available from: http://discovery.ucl.ac.uk/121079/

7. Nguyen NX, Weismann M, Trauzettel-Klosinski S. Improvement of reading speed after providing of low vision aids in patients with age-related macular degeneration. Acta Ophthalmol. 2009;87:849-853.

8. Jacewicz E, Fox RA, O’Neill C, Salmons J. Articulation rate across dialect, age, and gender. Lang Var Change [Internet]. 2009 July 8;21:233. Available from: http://www.journals.cambridge.org/abstract_S0954394509990093

9. Whiteside SP. Temporal-based acoustic-phonetic patterns in read speech: some evidence for speaker sex differences. J Int Phon Assoc [Internet]. 1996;26:23-40. Available from: http://www.journals.cambridge.org/abstract_S0025100300005302 


\section{IREST CANADIAN VALIDATION}

10. Statistics Canada. An increasingly diverse linguistic landscape : Highlights from the 2016 Census. Dly [Internet]. 2017. Available from: https://www150.statcan.gc.ca/n1/dailyquotidien/170817/dq170817a-eng.htm

11. Cop U, Drieghe D, Duyck W. Eye Movement Patterns in Natural Reading: A Comparison of Monolingual and Bilingual Reading of a Novel. PLoS One [Internet].

2015;10:e0134008. Available from: http://www.ncbi.nlm.nih.gov/pubmed/26287379

12. Gollan TH, Montoya RI, Cera C, Sandoval TC. More use almost always means a smaller frequency effect: Aging, bilingualism, and the weaker links hypothesis次. J Mem Lang [Internet]. 2008 April;58:787-814. Available from:

http://linkinghub.elsevier.com/retrieve/pii/S0749596X07000836

13. Favreau M, Segalowitz NS. Automatic and controlled processes in the first- and secondlanguage reading of fluent bilinguals. Mem Cognit [Internet]. 1983 November;11:565574. Available from: http://www.springerlink.com/index/10.3758/BF03198281

14. Lee J, Schallert DL. The Relative Contribution of L2 Language Proficiency and L1 Reading Ability to L2 Reading Performance: A Test of the Threshold Hypothesis in an EFL Context. TESOL Q [Internet]. 1997 January;31:713. Available from: http://www.jstor.org/stable/3587757?origin=crossref

15. Segalowitz N, Hulstijn J. Automaticity in bilingualism and second language learning. In: Kroll JF, De Groot AMB, editors. Handbook of bilingualism: Psycholinguistic approaches. Oxford: Oxford University Press; 2005. pp 371-388.

16. Canadian Institutes of Health Research, Natural Sciences and Engineering Research Council of Canada, Social Sciences and Humanities Research Council of Canada. TriCouncil Policy Statement: Ethical conduct for research involving humans. 2014. Available 


\section{IREST CANADIAN VALIDATION}

from:

http://www.pre.ethics.gc.ca/pdf/eng/tcps2/TCPS_2_FINAL_Web.pdf\%0Ahttp://www.pre. ethics.gc.ca\%0Ahttp://www.pre.ethics.gc.ca/pdf/eng/tcps2-

2014/TCPS_2_FINAL_Web.pdf

17. Bach M. The Freiburg Visual Acuity Test-Automatic Measurement of Visual Acuity. Optom Vis Sci. 1996;73:49-53.

18. Kurtenbach A, Langrová H, Messias A, Zrenner E, Jägle H. A comparison of the performance of three visual evoked potential-based methods to estimate visual acuity. Doc Ophthalmol [Internet]. 2013 February 11 [cited 2017 February 24];126:45-56. Available from: http://link.springer.com/10.1007/s10633-012-9359-5

19. Schulze-Bonsel K, Feltgen N, Burau H, Hansen L, Bach M. Visual Acuities "Hand Motion" and "Counting Fingers" Can Be Quantified with the Freiburg Visual Acuity Test. Investig Opthalmology Vis Sci [Internet]. 2006 March 1 [cited 2017 February 24];47:1236. Available from:

http://iovs.arvojournals.org/article.aspx?doi=10.1167/iovs.05-0981

20. Dienes Z. Bayesian Versus Orthodox Statistics: Which Side Are You On? Perspect Psychol Sci [Internet]. 2011 May;6:274-290. Available from:

http://dx.doi.org/10.1177/1745691611406920\%5Cnhttp://pps.sagepub.com/lookup/doi/10. $1177 / 1745691611406920$

21. Wetzels R, Matzke D, Lee MD, Rouder JN, Iverson GJ, Wagenmakers E-J. Statistical Evidence in Experimental Psychology. Perspect Psychol Sci [Internet]. 2011 May;6:291298. Available from: http://journals.sagepub.com/doi/10.1177/1745691611406923

22. The jamovi project. Jamovi 1.0. 2019. 


\section{IREST CANADIAN VALIDATION}

23. Owsley C, McGwin G, Lee PP, Wasserman N, Searcey K. Characteristics of low-vision rehabilitation services in the United States. Arch Ophthalmol (Chicago, Ill 1960) [Internet]. 2009 May;127:681-9. Available from: http://archopht.amaassn.org/cgi/reprint/127/5/681\%5Cnhttp://0ovidsp.ovid.com.wam.city.ac.uk/ovidweb.cgi?T=JS\&PAGE=reference \&D=emed9\&NEW $\mathrm{S}=\mathrm{N} \& \mathrm{AN}=2009256469$

24. Binns AM, Bunce C, Dickinson C, Harper R, Tudor-Edwards R, Woodhouse M, Linck P, Suttie A, Jackson J, Lindsay J, et al. How effective is low vision service provision? A systematic review. Surv Ophthalmol [Internet]. 2012;57:34-65. Available from: http://dx.doi.org/10.1016/j.survophthal.2011.06.006

25. Robinson S. Advanced Glaucoma and Low Vision: Evaluation and Treatment. In: Schacknow PN, Samples JR, editors. The Glaucoma Book: A Practical, Evidence-Based Approach to Patient Care. New York, NY: Springer New York; 2010. pp 351-381. Available from: http://link.springer.com/10.1007/978-0-387-76700-0

26. Zimmerman G, Zebehazy K, Moon M. Optics and low vision devices. In: Corn AL, Erin $\mathrm{JN}$, editors. Foundations of low vision : clinical and functional perspectives. 2nd ed. New York: AFB; 2010. pp 192-237.

27. Carver RP. Reading Rate: Theory, Research, and Practical Implications. J Read [Internet]. 1992;36:84-95. Available from: http://www.jstor.org/stable/40016440

28. Smith SG, Curtis LM, O’Conor R, Federman AD, Wolf MS. ABCs or 123s? The independent contributions of literacy and numeracy skills on health task performance among older adults. Patient Educ Couns [Internet]. 2015 August;98:991-7. Available from: http://dx.doi.org/10.1016/j.pec.2015.04.007 


\section{IREST CANADIAN VALIDATION}

29. Wolf MS, Gazmararian JA, Baker DW. Health literacy and functional health status among older adults. Arch Intern Med [Internet]. 2005 September 26;165:1946-52. Available from: http:/www.ncbi.nlm.nih.gov/pubmed/16186463

30. Kobayashi LC, Smith SG, O’Conor R, Curtis LM, Park D, von Wagner C, Deary IJ, Wolf MS. The role of cognitive function in the relationship between age and health literacy: a cross-sectional analysis of older adults in Chicago, USA. BMJ Open [Internet]. 2015 April 23;5:e007222. Available from: http://www.ncbi.nlm.nih.gov/pubmed/25908675

31. Elliott DB, Bullimore MA, Patla AE, Whitaker D. Effect of a cataract simulation on clinical and real world vision. Br J Ophthalmol [Internet]. 1996 September 1 [cited 2017 February 24];80:799-804. Available from:

http://bjo.bmj.com/cgi/doi/10.1136/bjo.80.9.799

32. Gao X, Loomes M. A new approach to image enhancement for the visually impaired. Electron Imaging [Internet]. 2016 February 14;2016:1-7. Available from: http://www.ingentaconnect.com/content/10.2352/ISSN.2470-1173.2016.20.COLOR-325

33. Rousek JB, Hallbeck MS. Improving and analyzing signage within a healthcare setting. Appl Ergon [Internet]. 2011 November;42:771-784. Available from:

http://linkinghub.elsevier.com/retrieve/pii/S0003687011000032

34. Crossland MD, Culham LE, Rubin GS. Fixation stability and reading speed in patients with newly developed macular disease*. Ophthalmic Physiol Opt [Internet]. 2004 July [cited 2017 February 24];24:327-333. Available from:

http://doi.wiley.com/10.1111/j.1475-1313.2004.00213.x

35. Nguyen NX, Stockum A, Hahn GA, Trauzettel-Klosinski S. Training to improve reading speed in patients with juvenile macular dystrophy: a randomized study comparing two 


\section{IREST CANADIAN VALIDATION}

training methods. Acta Ophthalmol [Internet]. 2011 February [cited 2017 February

24];89:e82-e88. Available from: http://doi.wiley.com/10.1111/j.1755-3768.2010.02081.x

36. Fork in the Road Vision Rehabilitation Services. Fork in the Road. [cited 2017 February 24]. Available from: https://www.lowvisionsimulators.com/

37. Regie de l'assurance maladie du Quebec/RAMQ. Services covered in Quebec - Visual devices. 2006;2010.

38. Coleman HR, Chan C-C, Ferris FL, Chew EY. Age-related macular degeneration. Lancet (London, England) [Internet]. 2008 November 22;372:1835-45. Available from:

http://www.ncbi.nlm.nih.gov/pubmed/19027484\%0Ahttp://www.pubmedcentral.nih.gov/a rticlerender.fcgi?artid=PMC2603424

39. Cheong AMY, Legge GE, Lawrence MG, Cheung SH, Ruff M a. Relationship between slow visual processing and reading speed in people with macular degeneration. Vision Res. 2007;47:2943-2955.

40. Crossland MD, Rubin GS. Eye movements and reading in macular disease: further support for the shrinking perceptual span hypothesis. Vision Res [Internet]. 2006 February;46:590-7. Available from: http://www.ncbi.nlm.nih.gov/pubmed/16005930

41. Cerulli A, Cesareo M, Ciuffoletti E, Montanaro ML, Mancino R, Mirisola C, Sorge R, Cedrone C, Nucci C, Cerulli L. Evaluation of eye movements pattern during reading process in patients with glaucoma: A microperimeter study. Eur J Ophthalmol. 2014.

42. Ishii M, Seki M, Harigai R, Abe H, Fukuchi T. Reading performance in patients with glaucoma evaluated using the MNREAD charts. Jpn J Ophthalmol. 2013.

43. Ramulu PY, West SK, Munoz B, Jampel HD, Friedman DS. Glaucoma and reading speed: The salisbury eye evaluation project. Arch Ophthalmol. 2009. 
IREST CANADIAN VALIDATION

Table 1.

Participant's Self-Reported Language Fluency

\begin{tabular}{|c|c|c|c|c|c|c|}
\hline & $n$ & No Ability & Elementary & Moderate & Very Good & Fluent \\
\hline \multicolumn{7}{|l|}{ Study 1} \\
\hline English & 25 & & & & & \\
\hline Speaking & & 0 & 0 & 0 & 1 & 24 \\
\hline Reading & & 0 & 0 & 0 & 2 & 23 \\
\hline Writing & & 0 & 0 & 0 & 4 & 21 \\
\hline Listening & & 0 & 0 & 0 & 1 & 24 \\
\hline French & 24 & & & & & \\
\hline Speaking & & 1 & 4 & 8 & 10 & 1 \\
\hline Reading & & 1 & 2 & 5 & 12 & 4 \\
\hline Writing & & 2 & 5 & 10 & 7 & 0 \\
\hline Listening & & 1 & 2 & 6 & 8 & 7 \\
\hline Other & 15 & & & & & \\
\hline Speaking & & 1 & 2 & 6 & 4 & 2 \\
\hline Reading & & 2 & 3 & 2 & 6 & 2 \\
\hline Writing & & 4 & 3 & 7 & 0 & 1 \\
\hline Listening & & 2 & 0 & 4 & 4 & 5 \\
\hline \multicolumn{7}{|l|}{ Study 2} \\
\hline English & 50 & & & & & \\
\hline Speaking & & 0 & 0 & 0 & 3 & 47 \\
\hline Reading & & 0 & 0 & 0 & 5 & 45 \\
\hline Writing & & 0 & 0 & 1 & 7 & 42 \\
\hline Listening & & 0 & 0 & 0 & 2 & 48 \\
\hline French & 50 & & & & & \\
\hline Speaking & & 2 & 8 & 17 & 16 & 7 \\
\hline Reading & & 1 & 4 & 12 & 24 & 9 \\
\hline Writing & & 5 & 11 & 18 & 10 & 6 \\
\hline Listening & & 3 & 5 & 8 & 18 & 16 \\
\hline Other & 27 & & & & & \\
\hline Speaking & & 0 & 3 & 17 & 4 & 3 \\
\hline Reading & & 7 & 4 & 7 & 6 & 3 \\
\hline Writing & & 7 & 9 & 7 & 3 & 1 \\
\hline Listening & & 1 & 3 & 10 & 5 & 8 \\
\hline
\end{tabular}

Note. Of the 42 participants with third languages, these languages included: Arabic, Dutch, Farsi, French, German, Hebrew, Hindu, Italian, Moroccan, Polish, Punjabi, Spanish, Tagalog, and Tamil. 


\section{IREST CANADIAN VALIDATION}

Table 2.

Results of the One Sample t-tests Between Reading Speeds of UK and Canadian Samples on the IReST.

\begin{tabular}{|c|c|c|c|c|c|c|c|c|c|c|c|c|c|}
\hline \multirow[t]{2}{*}{ IReST } & \multirow[b]{2}{*}{$\mathrm{n}$} & \multirow{2}{*}{$\begin{array}{c}\text { UK } \\
\text { Sample } \\
m \\
\end{array}$} & \multicolumn{2}{|c|}{ Canadian Sample } & \multirow[b]{2}{*}{ Mdiff } & \multicolumn{8}{|c|}{ One Sample t-tests } \\
\hline & & & $m$ & $s d$ & & $d f$ & $t$ & $p$ & $95 \% C I$ & Cohen's d & $95 \% C I$ & $B F_{10}$ & Error \% \\
\hline 1 & 25 & 236 & 230.70 & 35.23 & -5.30 & 24 & -0.75 & 0.22 & {$[-17.36,6.75]$} & -0.15 & {$[-0.48,0.18]$} & 0.41 & $6.27 \times 10^{5}$ \\
\hline 2 & 25 & 243 & 243.71 & 42.10 & 0.71 & 24 & 0.08 & 0.53 & {$[-13.69,15.1]$} & 0.02 & {$[-0.31,0.34]$} & 0.20 & $7.55 \times 10^{-5}$ \\
\hline 3 & 25 & 227 & 238.44 & 41.86 & 11.44 & 24 & 1.37 & 0.90 & {$[-2.92,25.8]$} & 0.27 & {$[-0.06,0.60]$} & 0.09 & $1.37 \times 10^{-6}$ \\
\hline 4 & 25 & 244 & 243.06 & 39.67 & -0.94 & 24 & -0.11 & 0.45 & {$[-14.48,12.6]$} & -0.02 & {$[-0.35,0.30]$} & 0.23 & $2.74 \times 10^{-4}$ \\
\hline 5 & 25 & 229 & 239.02 & 38.74 & 10.02 & 24 & 1.29 & 0.89 & {$[-3.27,23.3]$} & 0.25 & {$[-0.07,0.59]$} & 0.10 & $4.87 \times 10^{-6}$ \\
\hline 6 & 25 & 197 & 201.31 & 32.85 & 4.31 & 24 & 0.66 & 0.74 & {$[-6.88,15.5]$} & 0.13 & {$[-0.20,0.46]$} & 0.14 & $8.76 \times 10^{-5}$ \\
\hline 7 & 25 & 232 & 230.82 & 39.58 & -1.18 & 24 & -0.14 & 0.44 & {$[-14.75,12.4]$} & -0.20 & {$[-0.35,0.30]$} & 0.24 & $3.09 \times 10^{-4}$ \\
\hline 8 & 25 & 237 & 236.68 & 42.07 & -0.32 & 24 & -0.03 & 0.48 & {$[-14.74,14.1]$} & -0.01 & {$[-0.33,0.32]$} & 0.22 & $1.84 \times 10^{-4}$ \\
\hline 9 & 25 & 226 & 233.73 & 44.83 & 7.73 & 24 & 0.86 & 0.80 & {$[-7.64,23.1]$} & 0.17 & {$[-0.16,0.50]$} & 0.12 & $6.40 \times 10^{-5}$ \\
\hline 10 & 25 & 211 & 210.34 & 31.80 & -0.66 & 24 & -0.10 & 0.45 & {$[-11.53,10.2]$} & -0.02 & {$[-0.35,0.30]$} & 0.23 & $2.58 \times 10^{-4}$ \\
\hline
\end{tabular}




\section{IREST CANADIAN VALIDATION}

Table 3.

Mean Reading Speed Across Conditions

\begin{tabular}{ccccccc}
\hline Condition & M & SD & Mdiff $^{\dagger}$ & N & Min & Max \\
\hline CS & 230.78 & 36.68 & - & 25.00 & 141.04 & 331.56 \\
RC & 205.39 & 30.59 & -25.39 & 50.00 & 130.81 & 295.94 \\
RCI & 171.39 & 41.03 & -59.39 & 50.00 & 53.89 & 278.79 \\
\hline
\end{tabular}

Note. Mean reading speeds in words per minute (WPM) across all 10 IReST in the Canadian Sample (CS), the Reading Comprehension (RC) condition, and the Reading Comprehension with Impairment (RCI) condition.

${ }^{\dagger}$ Mean difference between the RC/RCI conditions and the CS condition. 
Table 4.

Descriptive statistics for the IReST across conditions

\begin{tabular}{|c|c|c|c|c|c|c|c|c|c|c|}
\hline Condition & IReST 1 & IReST 2 & IReST 3 & IReST 4 & IReST 5 & IReST 6 & IReST 7 & IReST 8 & IReST 9 & IReST 10 \\
\hline \multicolumn{11}{|c|}{ Canadian Sample (CS) - Study 1} \\
\hline Mean & 230.70 & 243.71 & 238.44 & 243.06 & 239.02 & 201.31 & 230.82 & 236.68 & 233.73 & 210.34 \\
\hline SD & 35.23 & 42.10 & 41.86 & 39.67 & 38.74 & 32.85 & 39.58 & 42.07 & 44.83 & 31.80 \\
\hline $\mathrm{N}$ & 25.00 & 25.00 & 25.00 & 25.00 & 25.00 & 25.00 & 25.00 & 25.00 & 25.00 & 25.00 \\
\hline Min & 171.96 & 169.15 & 177.32 & 178.20 & 169.69 & 141.04 & 157.39 & 146.11 & 159.06 & 150.46 \\
\hline Max & 299.71 & 329.33 & 331.56 & 319.87 & 324.49 & 269.28 & 305.98 & 312.77 & 317.48 & 282.66 \\
\hline \multicolumn{11}{|c|}{ Normal Vision - Reading Comprehension (RC) - Study 2} \\
\hline Mean & 208.74 & 220.26 & 213.62 & 222.59 & 214.65 & 181.34 & 200.09 & 208.52 & 199.00 & 185.05 \\
\hline SD & 34.81 & 34.04 & 29.80 & 32.87 & 29.15 & 22.04 & 35.87 & 33.62 & 30.78 & 28.36 \\
\hline $\mathrm{N}^{\dagger}$ & 25.00 & 25.00 & 25.00 & 25.00 & 25.00 & 25.00 & 25.00 & 25.00 & 25.00 & 25.00 \\
\hline Min & 144.57 & 142.96 & 139.43 & 155.66 & 156.09 & 144.70 & 130.81 & 159.29 & 138.48 & 133.20 \\
\hline Max & 266.82 & 277.11 & 253.02 & 295.94 & 267.83 & 226.51 & 271.24 & 285.03 & 263.66 & 242.34 \\
\hline \multicolumn{11}{|c|}{ Simulated Impairment - Reading Comprehension (RCI) - Study 2} \\
\hline Mean & 166.01 & 167.45 & 174.63 & 168.13 & 172.46 & 154.48 & 178.62 & 186.77 & 176.48 & 168.89 \\
\hline SD & 49.05 & 51.90 & 46.08 & 48.46 & 46.47 & 31.05 & 32.89 & 38.79 & 39.64 & 37.29 \\
\hline $\mathrm{N}^{\dagger}$ & 25.00 & 25.00 & 25.00 & 25.00 & 25.00 & 25.00 & 25.00 & 25.00 & 25.00 & 25.00 \\
\hline Min & 53.89 & 57.56 & 77.80 & 57.11 & 60.15 & 96.06 & 116.96 & 90.44 & 56.83 & 57.16 \\
\hline Max & 249.18 & 278.79 & 254.65 & 245.98 & 256.76 & 223.40 & 243.14 & 264.56 & 248.74 & 241.68 \\
\hline
\end{tabular}

Note. Mean reading speeds for each IReST for the condition.

${ }^{\dagger}$ Fifty individuals took part in Study 2, however, participants read 5 texts from the IReST with their normal vision and 5 texts from the IReST with a simulated visual impairment in a counterbalanced order of text presentation and condition. Therefore there are only 25 individuals per text, per condition. 


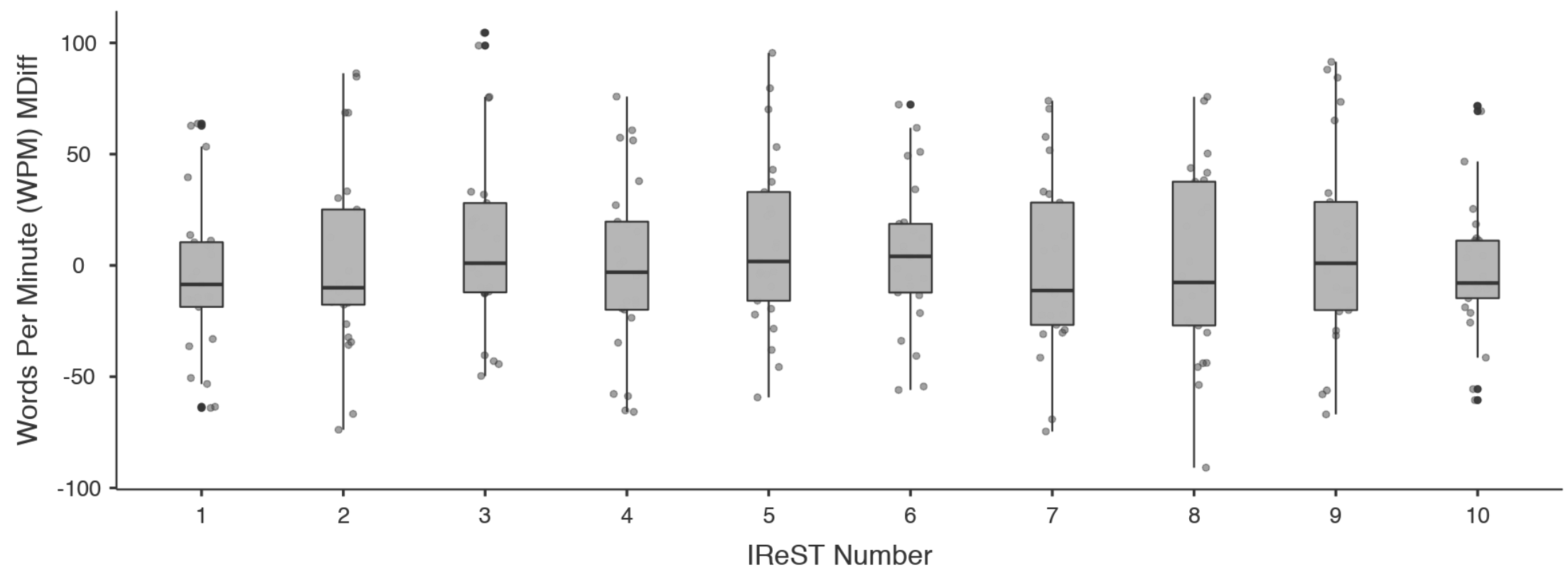

Figure 1. Mean difference between Canadian and UK reading speeds on the IReST. Boxplot of mean difference in reading speeds in words per minute (WPM) between the Canadian and UK English samples. 


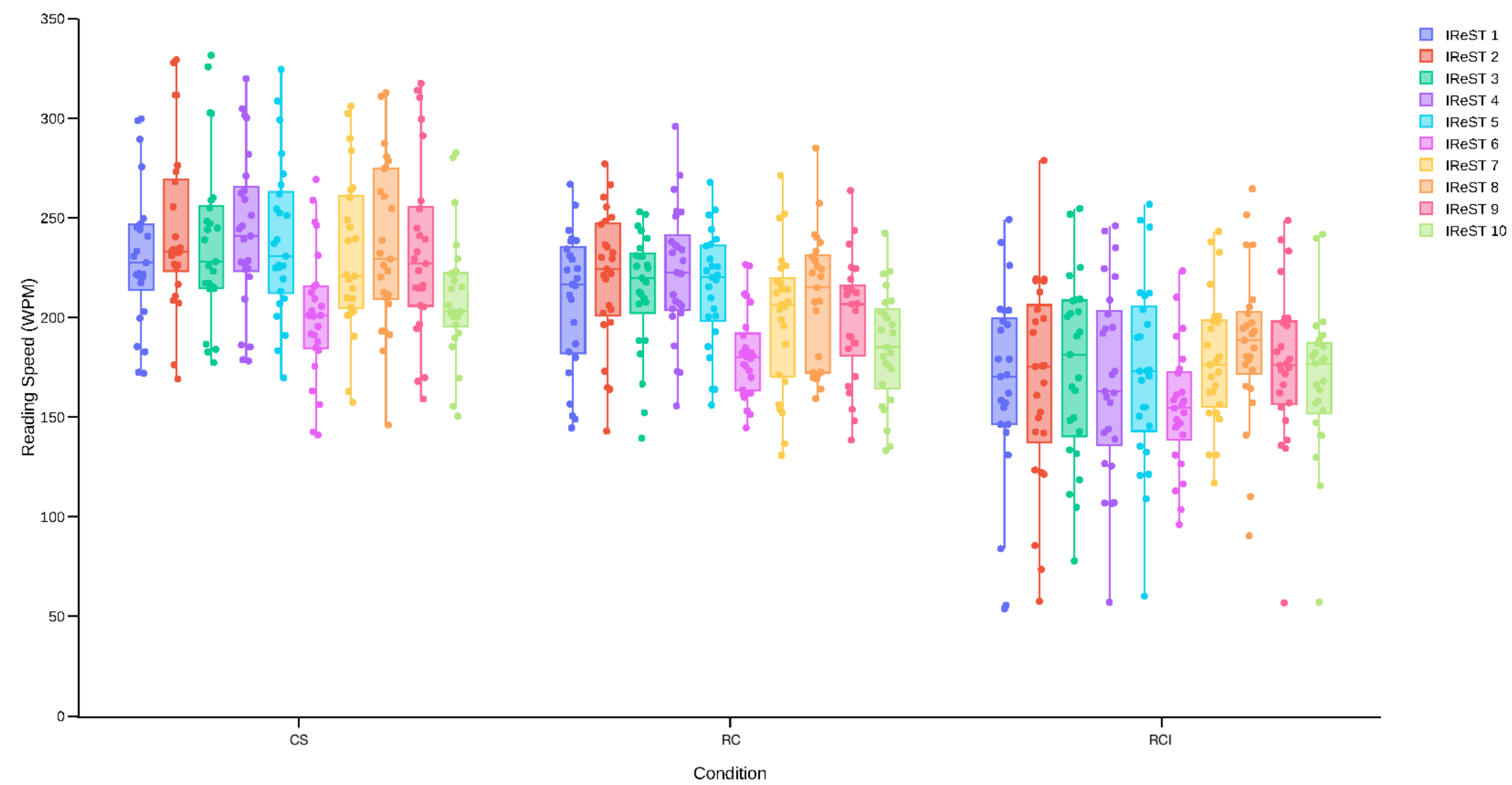

Figure 2. Comparison of Reading Speed on the IReST. Differences in mean reading speeds in words per minute (WPM) between

the Canadian Sample (CS), the Reading Comprehension condition (RC), and the Reading Comprehension with Impairment condition $(\mathrm{RCI})$. 